\title{
Vitamin A, Vitamin E, Lutein and $\beta$-Carotene in Lung Tissues from Subjects with Chronic Obstructive Pulmonary Disease and Emphysema
}

\author{
Michael W. Schäffer, Somdutta Sinha Roy, Shyamali Mukherjee, Salil K. Das* \\ Department of Biochemistry and Cancer Biology, Meharry Medical College, Nashville, USA \\ Email: ${ }^{*}$ sdas@mmc.edu
}

Received March 2, 2013; revised April 3, 2013; accepted April 10, 2013

Copyright (C) 2013 Michael W. Schäffer et al. This is an open access article distributed under the Creative Commons Attribution License, which permits unrestricted use, distribution, and reproduction in any medium, provided the original work is properly cited.

\begin{abstract}
Vitamin A (VA) and its active metabolites play an essential role in lung airway function. Patients with moderate to severe chronic obstructive pulmonary disease (COPD) have a lower serum retinol concentration, and improvement of their 1-second Forced Expiratory Volume (FEV1) is achieved with VA supplementation. In order to test our hypothesis that the VA signaling pathway is compromised in COPD, we obtained 20 lung samples from COPD patients differing in the degree of emphysema as judged by their FEV\% values. All were smokers or were exposed to secondhand smoke. Levels of VA (retinol/retinyl ester), tocopherols and carotenoids (lutein, beta-carotene) in these samples were determined using HPLC. Additional analytes beside VA were included for their known roles as antioxidants and modulators of VA-action. VA levels (retinol/retinyl ester) decreased significantly with the increase in severity of emphysema. Among other analytes, $\alpha$-tocopherol levels fell by $25.8 \%$ in the severe emphysema group in comparison to the mild emphysema group, and lutein levels similarly decreased in severe compared to moderate emphysema groups. However, beta-carotene levels remained unchanged. Thus there is a significant linear correlation between lung VA-levels and the severity of emphysema. There was also a significant reduction in the levels of $\alpha$-, $\delta$-tocopherol and lutein in the severe emphysema group of COPD patients who either smoked or were exposed to smoke.
\end{abstract}

Keywords: COPD; $\beta$-Carotene; Emphysema; Lutein; Vitamin A; Vitamin E; HPLC

\section{Introduction}

Chronic bronchitis and emphysema present two types of COPD. Presently, COPD is the fourth leading killer of adults in the US and likely to be the third leading cause of death by 2020 [1]. Approximately 12 million adults are diagnosed with COPD, 120,000 die from it each year, and an additional 12 million adults may have undiagnosed COPD [2]. As an added problem, smokers with COPD have a higher risk of lung cancer [3].

Previous studies suggested a link between a local respiratory vitamin A deficiency and the severity of COPD [4]. The Second National Health and Nutrition Examination Survey [5] documented that a smoker's risk of developing COPD is dose-dependently decreased by the intake of vitamin A. Vitamin A and its active metabolite all-trans retinoic acid (Tretinoin, ATRA) are important for growth and differentiation of many tissues/organs, including lung $[6,7]$. Further, retinoid signaling elements,

\footnotetext{
*Corresponding author.
}

such as receptors and binding proteins have been described in the adult lung [7]. Numerous findings suggest that ATRA may protect against emphysema [8,9]. Recently, several studies have shown that ATRA is effective in promoting alveolization in papain-induced emphysema in dogs [10] and elastase-induced emphysema in adult rats [11].

We previously reported that exposure of guinea pigs to cigarette smoke caused an accumulation of lung retinol and a decrease in ATRA [12], suggesting an abnormality in retinoid metabolism and signaling. These findings raised the question as to whether smoke-associated COPD similarly result in abnormalities in retinoid metabolism and signaling by altering intracellular levels of vitamin A, and as a consequence ATRA, its active metabolite.

Tocopherols (vitamin E) are a group of essential micronutrients that act mainly as lipophilic antioxidants which protect polyunsaturated fatty acids from oxidative damage, thus maintaining fluidity and integrity of cellu- 
lar membranes [13]. Since smokers subject their lungs to an increased burden of oxidative damage, a reduction in lung tocopherol-levels could be a consequence that contributes to the degree of severity of COPD. Although a systemic vitamin E deficiency is normally unseen in the Western world, circulating levels of vitamin $\mathrm{E}$ can be reduced in premature infants with bronchopulmonary dysplasia who are also selenium deficient [14].

Lutein, a dietary carotenoid that serves as a powerful antioxidant, plays an important role in ocular health and in the prevention of cardiovascular disease, stroke and lung cancer [15]. In previous studies we showed that lutein accumulates in guinea pig [16] and human lungs [17]. Although not much is known about the specific role of lutein in lung, a report [18] identified $\beta$-cryptoxanthin and lutein as natural ligands for the nuclear retinoic acid receptors (RAR) - especially RAR $\alpha$ and RAR $\gamma$. The carotenoids $\beta$-carotene and lycopene failed to show a similar activity.

Although the role of dietary antioxidant $\beta$-carotene remains controversial [19]; an epidemiological study reported an inverse correlation between serum $\beta$-carotene and the incidence of lung cancer [20]. In contrast, two intervention trials (ATCB, CARET) showed that high doses of $\beta$-carotene given to smokers actually raised rather than lower their chances of developing lung cancer $[21,22]$. $\beta$-Carotene like lutein is deposited in human lung tissue [17].

We hypothesized that there is a lung deficiency of vitamin A and vitamin E and some carotenoids in COPD patients with emphysema, and further that there is a relationship between the severity of emphysema and the levels of these nutrients. Dietary carotenoids were included because of their roles as potential effectors on the vitamin A-signaling pathway and contributors to the antioxidant capacity of the cells.

We therefore analyzed lung tissues from 20 COPD patients classified as having mild, moderate or severe emphysema.

\section{Materials and Methods}

Human lung samples, preparation/extraction. Frozen human lung samples were obtained from the Lung Tissue Research Consortium (LTRC) of the National Heart, Lung, and Blood Institute, Bethesda, MD. Until extraction for analysis, samples were stored at $-80^{\circ} \mathrm{C}$. The LTRC provided us with de-identified information regarding lung tissue donors including age, gender, smoking habits, FEV1\% values, and the location from which the samples were taken. The characteristics of the human subjects whose biopsies were used for HPLC-analyses are described in Table 1. Nine subjects were categorized as having "severe emphysema" with FEV1\% value $<50$ (average age was $52.6 \pm 6.7$ years), 8 subjects were cate-
Table 1. Characteristics of subjects.

\begin{tabular}{cccccc}
\hline Sample & & & & & Tissue \\
\hline ID & Gender & Age & FEV1\% & Smoking & Origin $^{*}$ \\
$\mathbf{1 9 2 3 6 1}$ & F & 50 & 12 & yes & RM \\
$\mathbf{2 4 2 4 2 0}$ & M & 63 & 14 & yes & LL \\
$\mathbf{2 9 4 9 4 5}$ & F & 52 & 15 & yes & RM \\
$\mathbf{1 2 2 5 4 1}$ & F & 55 & 19 & yes & RM \\
$\mathbf{1 5 7 4 2 1}$ & M & 46 & 20 & yes & RM \\
$\mathbf{1 2 8 6 1}$ & M & 51 & 22 & yes & LL \\
$\mathbf{1 3 2 6 2 5}$ & F & 63 & 24 & yes & RM \\
$\mathbf{2 9 5 1 6 7}$ & M & 49 & 27 & yes & LL \\
$\mathbf{1 2 6 5 7 1}$ & M & 44 & 40 & yes & RM \\
$\mathbf{2 0 0 9 3 0}$ & F & 78 & 52 & yes & RL \\
$\mathbf{2 6 4 5 8}$ & M & 73 & 54 & yes & RL \\
$\mathbf{1 8 3 5 6 1}$ & M & 83 & 57 & yes & RU \\
$\mathbf{2 7 1 8 5 1}$ & M & 77 & 68 & yes & LU \\
$\mathbf{1 4 4 4 6 2}$ & F & 73 & 69 & yes & RM \\
$\mathbf{1 5 8 2 3 1}$ & F & 61 & 72 & yes & LU \\
$\mathbf{1 7 8 9 6 7}$ & F & 72 & 72 & yes & RL \\
$\mathbf{3 0 7 5 3}$ & M & 75 & 77 & no ${ }^{\dagger}$ & LL \\
$\mathbf{2 6 0 1 1 1}$ & F & 61 & 84 & yes & RM \\
$\mathbf{2 6 1 3 6 1}$ & F & 63 & 89 & yes & RL \\
$\mathbf{1 7 6 5 6 2}$ & M & 73 & 105 & yes & LLO \\
\hline
\end{tabular}

"Abbreviations: $\mathrm{M}=$ male; $\mathrm{F}=$ female; $\mathrm{LLO}=$ left lower; $\mathrm{LL}=$ left ling; $\mathrm{LU}$ $=$ left upper; $\mathrm{RL}=$ right lower; $\mathrm{RM}=$ right middle; $\mathrm{RU}=$ right upper . ${ }^{\dagger}$ Secondhand smoke; FEV1\%-range: $<50$ severe, $50-80$ moderate, $>80$ mild.

gorized as having "moderate emphysema" with FEV1\% values ranging between 50 - 80 (average age was $72.3 \pm$ 7.8 years), and 3 subjects were categorized as having "mild emphysema" with FEV1\% values $>80 \%$ (average age was $65.7 \pm 4.4$ years). The average age of all 20 subjects was $62.9 \pm 11.7$ years; $52.4 \%$ were male and $47.6 \%$ were female. Amongst these subjects, only one was a nonsmoker but was subjected to cigarette smoke at home.

HPLC analysis, chemicals and reagents. Chemical and reagent resources, and High Pressure Liquid Chromatography (HPLC)-analyses were carried out as described earlier [17]. On average $100 \mathrm{mg}$ of each lung was homogenized, extracted and analyzed by HPLC for the analytes of 1) retinol and their found storage forms retinyl palmitate (RP) and retinyl stearate (RS), 2) tocopherols $(\alpha, \beta / \gamma$ and $\delta$ ) and 3) carotenoids (lutein and $\beta$-carotene). Values are expressed as mean \pm standard error of the mean (SEM). Statistical analyses were performed using GraphPad Prism Version 5.04 (GraphPad software, San Diego, CA). Statistical significances $(P$ values) were calculated using an unpaired $t$-test (www. graphpad.com) to each of 8 analytes concentrations in 
mild vs. moderate ${ }^{* * *}$, moderate vs. severe ${ }^{* *}$ and mild vs. severe* .

\section{Results}

\subsection{HPLC Analysis}

Figure 1 provides overlaid chromatograms of two representative lung samples from a patient with either severe (lower trace) or moderate emphysema (upper trace) at three different wavelengths in order to detect retinoids (Figure 1(a)), tocopherols (Figure 1(b)) and carotenoids (Figure 1(c)).

\subsection{Vitamin A}

Samples were extracted and analyzed by HPLC for the analytes retinol $(\mathrm{ROH})$ and their found storage forms RP and RS. Values for the total vitamin A content were calculated by adding found values for ROH, RP and RS per sample, given as nmol retinol per gram lung tissue. The mean and the corresponding SEM for each analytes in each group (mild: $\mathrm{n}=3$; moderate: $\mathrm{n}=8$; severe: $\mathrm{n}=9$ ) was calculated and plotted as a bar graph in Figure 2(a). Results using an unpaired $t$-test for vitamin A metabolites showed that difference in $\mathrm{ROH}-$ levels between the mild and the severe group were significant $\left({ }^{*}\right)$. However, the changes in total vitamin A were significantly different not only between the mild and severe group, but also between the moderate and the severe group (data not shown). The differences between RP values for moderate vs severe group $\left(^{* *}\right)$ were highly significant $(P<0.005)$.

Since our collection of samples comprises a wide range of FEV $1 \%$ in patient values (Table 1), we plotted those values against total vitamin A concentrations (Figure 2(b)) in order to determine if there was a linear relationship. The resulting graph demonstrates a significant inverse relationship between the total vitamin A lung levels as a function of the severity of emphysema in those patients $\left(r^{2}=0.3035, P=0.0145\right)$. Amongst the determined vitamin A-analytes, only the data for retinol showed a similar behavior, although with a smaller correlation coefficient $\left(r^{2}=0.2651\right)$.

As an allover trend, vitamin A values tended to decrease with increasing severity of emphysema.

\subsection{Vitamin $E$}

Samples were extracted and analyzed by HPLC for their vitamin $\mathrm{E}$ content, which included analytes for $\alpha, \beta / \gamma$ and $\delta$-tocopherol. The mean and the corresponding SEM for each analytes in each group $(\alpha-, \beta / \gamma$-tocopherol: mild: $\mathrm{n}=$ 3 ; moderate: $\mathrm{n}=7$; severe: $\mathrm{n}=9 ; \gamma$-tocopherol: mild: $\mathrm{n}=$ 3; moderate: $\mathrm{n}=8$; severe: $\mathrm{n}=9$ ) was calculated and plotted as a bar graph in Figure 3. Results from the unpaired $t$-test for tocopherol analytes showed that only the

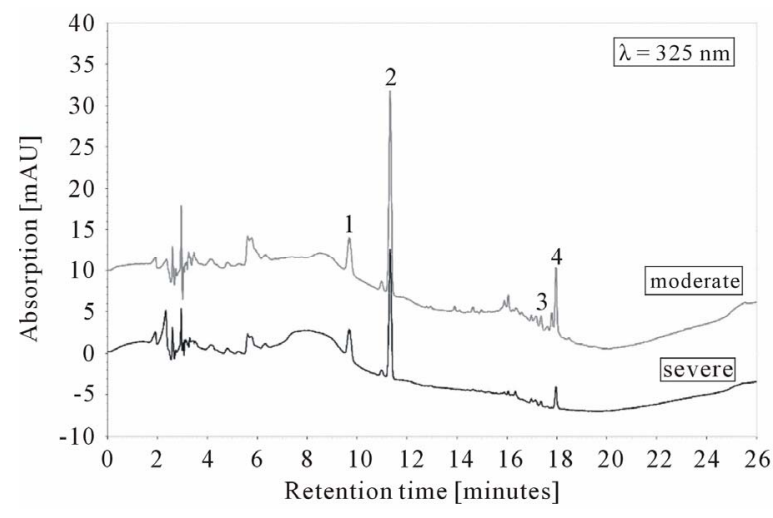

(a)

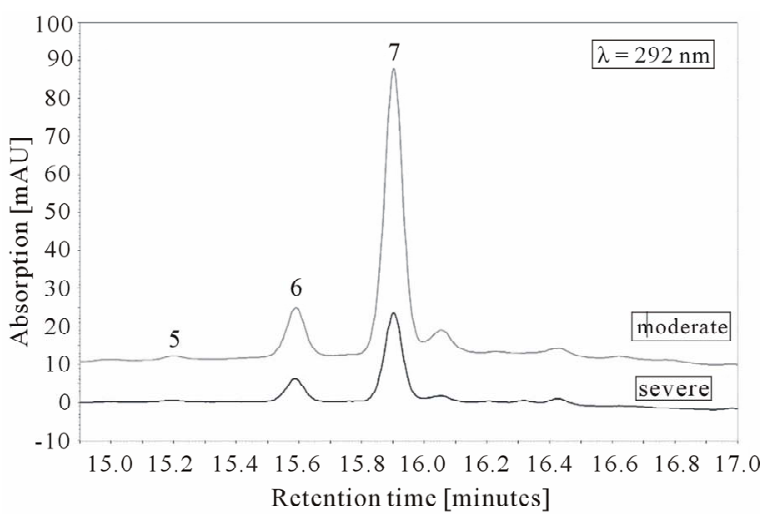

(b)

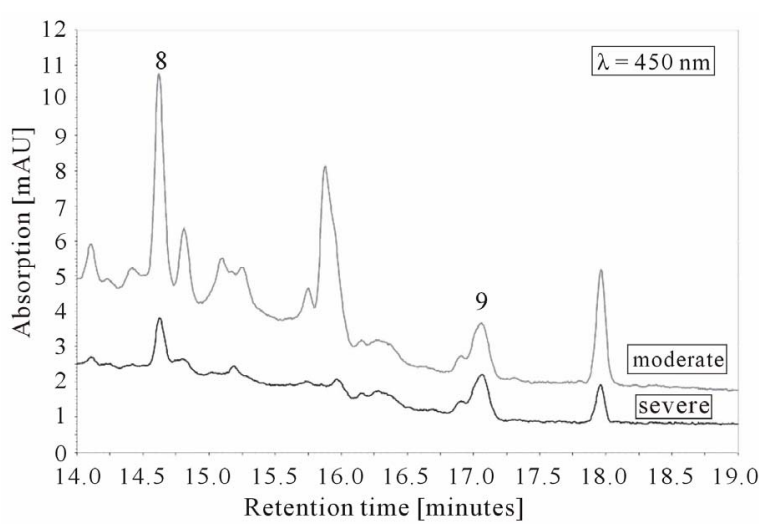

(c)

Figure 1. Chromatograms of two representative lung samples (upper: moderate, lower: severe) at different detection wavelengths. A: $\lambda=350 \mathrm{~nm}$ for vitamin A analytes; B: $\lambda=$ $292 \mathrm{~nm}$ for vitamin $E$ analytes; and $C: \lambda=450 \mathrm{~nm}$ for lutein and $\beta$-carotene. To allow for a direct comparison, the chromatograms were normalized to the same weight of lung sample. Numbers refer to each corresponding peak in both overlaid chromatograms. Peak assignments: (a) 1-all-trans retinol, 2-all-trans retinyl acetate (Internal Standard), 3all-trans retinyl palmitate, and 4-all-trans retinyl stearate; (b) 5- $\delta$-tocopherol, 6- $\beta / \gamma$-tocopherol, and 7- $\alpha$-tocopherol; (c) 8 -lutein, and 9- $\beta$-carotene. It should be noted that the applied separation method ${ }^{17}$ does not distinguish between the two isomers $\gamma$-tocopherol and $\beta$-tocopherol. Values will therefore represent always a sum of both analytes. 
Vitamin A

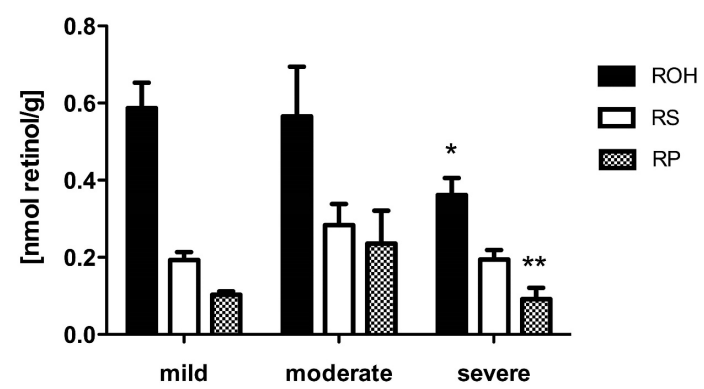

(a)

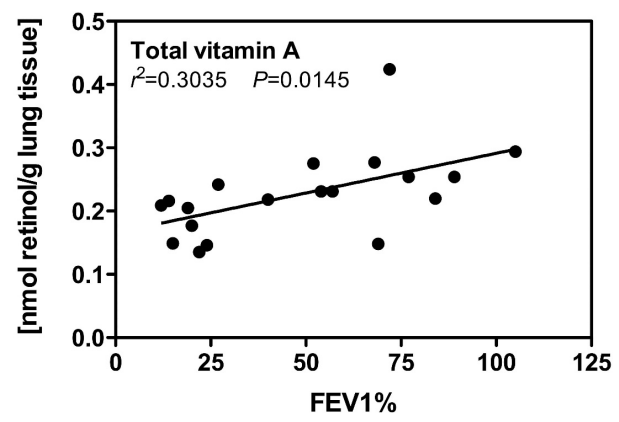

(b)

Figure 2. Vitamin A values (a) Bar graph of the mean and the corresponding SEM for the found values of retinol, retinyl palmitate and retinyl stearate, expressed in nmol retinol per gram wet weight lung tissue in each group (mild: $\mathbf{n}$ $=3$; moderate: $\mathrm{n}=8$; severe: $\mathrm{n}=9$ ). Significances $(P<0.05)$ from unpaired $t$-test are indicated by ${ }^{*}$ ) for mild vs. severe and **) for moderate vs. severe values; (b) Demonstration of an inverse linear correlation between the degree of emphysema, expressed in FEV1\%-values and the calculated amount of total vitamin $\mathrm{A}(=[\mathrm{ROH}]+[\mathrm{RP}]+[\mathrm{RS}])$ given in nmol retinol per gram wet weight lung tissue.

changes in $\delta$-tocopherol levels between the moderate and severe group $\left({ }^{* *}\right.$, Figure 3(c)) were statistically highly significant $(P<0.005)$. Although not statistically significant, there was a decrease of $25.8 \%$ in $\alpha$-tocopherol levels in the severe group compared to samples in the mild group (Figure 3(a)). Changes of $\beta / \gamma$-tocopherollevels between the groups were not significantly different from each other, however amongst the tested vitamin $\mathrm{E}$ analytes; it is the only one that showed increased levels with increasing severity, for e.g. the $\beta / \gamma$-tocopherol-levels in the severe group were $21.8 \%$ higher on average than in the mild group (Figure 3(b)).

\subsection{Carotenoids}

We also assayed for the carotenoids lutein and betacarotene. The mean and the corresponding SEM for the analytes lutein (mild: $\mathrm{n}=3$; moderate: $\mathrm{n}=7$; severe: $\mathrm{n}=$ 9) and beta-carotene (mild: $\mathrm{n}=3$, moderate: $\mathrm{n}=8$, severe: $\mathrm{n}=9$ ) in each group was calculated and plotted as a bar graph (Figure 4). The levels of beta-carotene between alpha-tocopherol

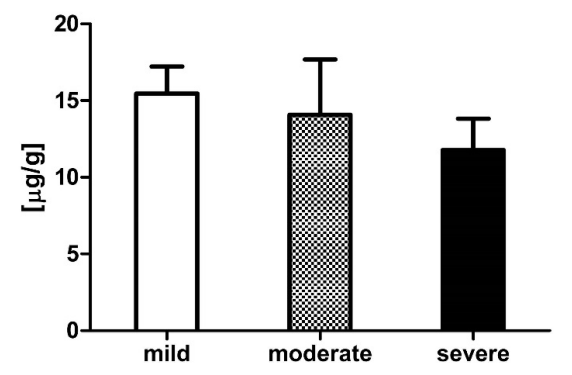

(a)

gamma/beta-tocopherol

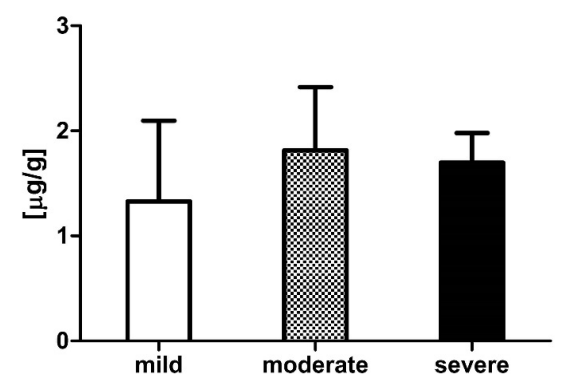

(b)

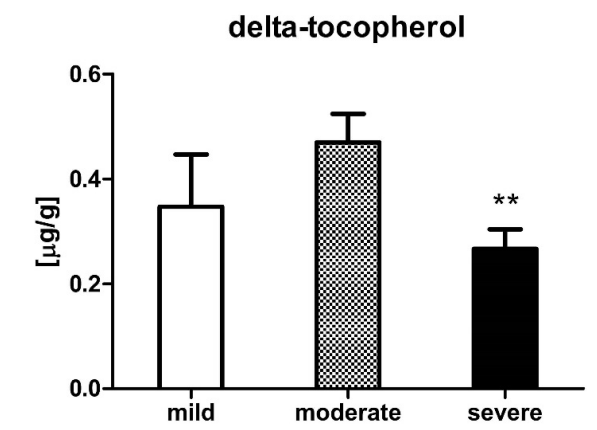

(c)

Figure 3. Vitamin $E$ values. Bar graph of the mean and the corresponding SEM for the found values of (a) $\alpha$-tocopherol; (b) $\beta / \gamma$-tocopherol and (c) $\delta$-tocopherol, expressed in g per gram wet weight lung tissue in each group $(\alpha-, \beta / \gamma$-tocopherol: mild: $n=3$; moderate: $n=7$; severe: $n=9 ; \delta$-tocopherol: mild: $n=2$; moderate: $n=8$; severe: $n=9$ ). Significances $(P<0.05)$ from unpaired $t$-test are indicated by $\left.{ }^{* * *}\right)$ for moderate vs. severe. It should be noted that $\alpha$-tocopherol-levels are found to be an order of magnitude higher than those for $\gamma / \beta$-tocopherol, which are again an order of magnitude higher than in the detected $\delta$-tocopherol-levels.

the groups did not change. In contrast, changes in lutein levels between the moderate and severe group were statistically significant $\left({ }^{* *}\right.$, Figure 4). Interestingly, lung tissue harvested from subjects in the moderate group accumulated almost twice the lutein than samples from the other two groups.

\section{Discussion}

There have been a number of human studies that have 


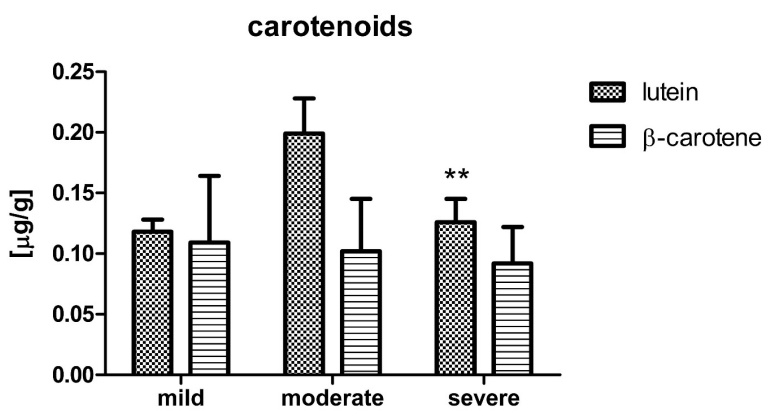

Figure 4. Carotenoid values. Bar graph of the mean and the corresponding SEM for the found values of lutein and $\beta$ carotene, expressed in $\mu \mathrm{g}$ per gram wet weight lung tissue in each (lutein: mild: $n=3$, moderate: $n=7$, severe: $n=9$; $\beta$-carotene: mild: $n=3$, moderate: $n=8$, severe: $n=9$ ), Significances $(P<0.05)$ from unpaired t-test are indicated by $^{* * *}$ ) for moderate vs. severe lutein values.

attempted to correlate intake of various micronutrients to the outcomes of COPD, asthma, bronchopulmonary dysplasia and lung cancer using either by nutritional surveys or blood chemistries with a variety of outcomes and conclusions [4,5]. They lacked, however, information about the concentration and composition of the analytes in the lung tissue, per se. To our knowledge, we report here for the first time the HPLC-analysis of micronutrients vitamins $\mathrm{A}$ and $\mathrm{E}$ and the carotenoids lutein and $\beta$-carotene in lung samples from human COPD subjects with varying degrees of emphysema.

In the case of vitamin A and its metabolites, earlier studies showed that the risk for COPD increased with decreasing serum vitamin A [23,24]; and that daily oral doses of vitamin A (25,000 IE) for 30 days attenuated the symptoms [25].

Although the overall homeostatic regulation of plasma retinol values was unaffected by the pathologic conditions of COPD, Paiva et al. [4] found a positive correlation between serum retinyl esters and FEV1/FVC-values in healthy smokers and in patients with moderate to severe COPD; again there was an improvement in pulmonary functions after vitamin A supplementation. Although smokers with COPD usually do not have a systemic vitamin A deficiency [4], a local deficiency may occur as a result of chronic exposure to cigarette smoke.

Our results show that lung vitamin A levels fell with the progression of disease, as judged by FEV1\% values. This local vitamin A deficiency may be caused by an increased demand for its active form ATRA, followed by a depletion of intra-cellular retinyl ester stores. We have found a highly statistically significant decrease in RP levels in the severe vs. moderate groups. This could be explained by an increased preference of retinyl ester hydrolase towards the palmitate-ester over the stearate-ester. Indeed, a report [26] showed that palmitate is the preferred fatty acyl moiety over stearate.
In addition, exposure to the environmental toxicant benzo [a] pyrene decreased the uptake of vitamin A into lung cells [27] and led to a local vitamin A deficiency in lung tissues [28]. The cellular uptake of retinol into target tissue is mainly mediated by the membrane-bound STRA6-receptor [29]. Since vitamin A is depleted in lungs of COPD-patients, ATRA-levels could also be significantly reduced. This is important because ATRA is required for the activation of STRA6-gene expression [29]. Thus, it seems likely that reduced STRA6-levels would aggravate a local vitamin A-deficiency. We are currently investigating this question in our laboratory.

Since a systemic supplementation may have severe toxic side effects, others have proposed the uptake of either vitamin A or ATRA in the lung by inhalation. Biesalski et al. [30] demonstrated that inhalation of an RPaerosol can successfully improve the vitamin A status of preschool children. Furthermore, Kohlhäufl et al. [31] reported a complete reversal of metaplasia/dysplasia in $44 \%$ (and partial remission in 12\%) of biopsies from smokers with premalignant lesions of the bronchial epithelium who were administered aerosolized vitamin A. Interestingly, a recent case study [32] on a 45-year-old patient with severe emphysema, a never smoker individual on a 6-month controlled ATRA-inhalation therapy, showed signs of improvement in daily life in terms of the hours spent without oxygen supplementation and a reduction in inflammatory markers such as serum IL-13.

Although our data do not reveal if the local vitamin A-deficiency is a cause or a consequence of COPD, a therapy with inhalable formulations of vitamin A may be beneficial for the alleviation of symptoms while avoiding potential side effects by administration of this vitamin. However a recent study demonstrated the increased susceptibility to the development of cigarette smoke-induced lung emphysema in mice with low but not deficient vitamin A status [33].

The most abundant tocopherol in the human lung, $\alpha$ tocopherol, fell $25.8 \%$ from values in the mild to severe groups. The reduced availability of this antioxidant may be scientifically important, since these patients face an increased oxidative stress burden due to cigarette smoke. In a recent case-control study with 1088 lung cancer patients and 1414 healthy matched controls, investigators [34] reported that an increased intake of dietary $\alpha$-tocopherol accounted for a $34 \%-53 \%$ reduction in lung cancer risk. Thus the found $25.8 \%$-reduction in $\alpha$-tocopherol levels in lungs in our study may be a critical risk factor for the development of lung cancer in COPD patients. Since $\alpha$-tocopherol inhibits retinyl ester hydrolase (REH) activity [35], the drop in $\alpha$-tocopherol-levels may also reduce its ability to inhibit REH, resulting in a reduction in RP.

There is emerging evidence that tocopherols in addi- 
tion to their antioxidant properties have other distinct functions, for example they can have direct and indirect effects on several enzymes involved in signal transduction [36] and anti-inflammatory effects [37]. Recent evidence suggested that $\delta$ - and $\alpha$-tocopherol and their metabolites are more potent inhibitors of cyclooxygenase- 2 (COX-2) than $\alpha$-tocopherol [37,38]. Interestingly, another report [39] showed that the expression of COX-2 in lungs of COPD patients with lung cancer was significantly increased compared to control subjects. This study together with our observation of finding decreased $\alpha$ - and $\delta$-tocopherol levels in these patients, suggest the involvement of low tocopherols and high COX-2 levels in COPD-related lung cancer.

Our findings that lutein levels significantly decrease whereas beta-carotene levels do not change cannot be simply explained by differences in dietary consumption. There is increasing evidence that the uptake, distribution and their function in the body are tightly regulated by different enzymes. Beta-carotene, for e.g. is transported primarily in very low-density lipoproteins (VLDL) and low-density lipoproteins (LDL), whereas lutein has a preference for high-density lipoproteins (HDL) [40]. In combination with specific cell surface receptors, the apolipoprotein components of the lipoproteins assist the carotenoids to their specific target tissue. Cellular LDLreceptors mediate beta-carotene uptake [40], whereas lutein uses class B scavenger receptors [41]. Interestingly, the steroidogenic acute regulatory domain 3 (StARD3) protein, also known as MLN64, has recently been identified as a lutein-binding protein in the human retinal macula [40]. Furthermore, StARD3 is involved in intra-cellular cholesterol transport [41]. Since StARD3 is expressed in many tissues including lung [42], we hypothesize that this protein may mediate lutein uptake in lung and when its function is compromised under significant oxidative stress, this leads to a reduction of lung lutein levels.

Our overall results suggest that with increasing severity of emphysema in COPD patients, the levels of retinol and total vitamin A (retinol + ester) are reduced in lung. Together with changes seen in lutein and $\alpha$ - and $\delta$-tocopherol, critical components of the cellular antioxidative defense have been reduced.

Among the COPD subjects included in this study, most were firsthand smokers, only one was secondhand smoker and none represented nonsmoker. Also there was no non-diseased control group. The limitation of the current study needs to be controlled by inclusion of these additional groups. It will be interesting to investigate whether blood levels of the nutrients of the COPD subjects vary with the degree of emphysema. Furthermore, we are currently investigating whether expression of certain mediators of retinoid action (trafficking, metabolism and signaling) in lung of COPD patients vary with the degree of emphysema.

\section{Acknowledgements}

This work was supported by NIH grant 1R03 HL095419$01 \mathrm{~A} 1$.

\section{REFERENCES}

[1] G. Veigi, A. Scognamiglio, S. Baldacci, F. Pistelli and L. Carrozzi, "Epidemiology of Chronic Obstructive Pulmonary Disease (COPD)," Respiration, Vol. 68, No. 1, 2001, pp. 4-19. doi: $10.1159 / 000050456$

[2] C. Roth, "Factsheet Chronic Obstructive Pulmonary Disease (COPD)," National Institutes of Health, Vol. 10, 2010. http://report.nih.gov/NIHfactsheets/Pdfs/ChronicObstruct ivePulmonaryDisease(NHLBI).pdf

[3] S. K. Das, "Harmful Effects of Cigarette Smoking," Molecular and Cellular Biochemistry, Vol. 253, No. 1, 2003, pp. 159-165. doi:10.1023/A:1026024829294

[4] S. A. Paiva, I. Godoy, H. Vannucchi, R. M. Favaro, R. R. Geraldo and A. O. Campana, "Assessment of Vitamin A status in Chronic Obstructive Pulmonary Disease Patients and Healthy Smokers," American Journal of Clinical Nutrition, Vol. 64, No. 6, 1996, pp. 928-934.

[5] A. McDowell, A. Engle, J. T. Massey and K. R. Maurer, "Plan and Operation of the Second Health and Nutrition Examination Survey, 1976-80," Vital and Health Statistics, Vol. 1, No. 15, 1981, pp. 81-1317.

[6] D. E. Ong and F. Chytil, "Changes in Levels of Cellular Retinol- and Retinoic-Acid-Binding Proteins of Liver and Lung during Perinatal Development of Rat," Proceedings of National Academy of Sciences USA, Vol. 73, No. 11, 1976, pp. 3976-3978. doi:10.1073/pnas.73.11.3976

[7] F. Chytil, "The Lungs and Vitamin A," American Journal of Physiology, Vol. 261, No. 5, 1992, pp. L517-527.

[8] N. R. MacIntyre, "Chronic Obstructive Pulmonary Disease: Emerging Medical Therapies," Respiratory Care, Vol. 49, No. 1, 2004, pp. 64-69.

[9] W. C. Bailey and D. P. Tashkin, "Pharmacologic Therapy: Novel Approaches for Chronic Obstructive Pulmonary Disease," Annals of the American Thoracic Society, Vol. 4, No. 7, 2007, pp. 543-548. doi:10.1513/pats.200701-017FM

[10] F. S. Rosenthal, "Aerosol Probes of Emphysema Progression in Dogs Treated with All-Trans Retinoic Acid-An Exploratory," Journal of Aerosol Medicine, Vol. 20, No. 2, 2007, pp. 83-96. doi:10.1089/jam.2007.0581

[11] G. D. Massaro and D. Massaro, "Retinoic Acid Treatment Abrogates Elastase-Induced Pulmonary Emphysema in Rats," Nature Medicine, Vol. 3, No. 6, 1997, pp. 675-677. doi:10.1038/nm0697-675

[12] S. Mukherjee, T. Nayyar, F. Chytil and S. K. Das, "Mainstream and Sidestream Cigarette Smoke Exposure Increases Retinol in Guinea Pig Lungs," Free Radical Biology and Medicine, Vol. 18, No. 3, 1995, pp. 507-514. doi:10.1016/0891-5849(94)00161-C 
[13] H. J. Nelis, E. D'Haese and K. Vermis, "Vitamin E," In: A. P. De Lenheer, W. E. Lambert and J. F. Van Bocxlaer, Eds., Modern Chromatographic Analysis of Vitamins, 3rd Edition, Marcel Dekker, New York, 2000, pp. 143-228.

[14] H. S. Falciglia, J. R. Johnson, J. Sullivan, et al., "Role of Antioxidant Nutrients and Lipid Peroxidation in Premature Infants with Respiratory Distress Syndrome and Bronchopulmonary Dysplasia," American Journal of Perinatology, Vol. 20, No. 2, 2003, pp. 97-107. doi:10.1055/s-2003-38315

[15] F. M. Painter, "Lutein and Zeaxanthin," Alternative Medicine Review, Vol. 10, No. 2, 2005, pp. 128-135.

[16] M. W. Schäffer, S. Sinha Roy, M. Mukherjee and S. K. Das, "Identification of Lutein, a Dietary Antioxidant Carotenoid in Guinea Pig Tissues," Biochemical and Biophysical Research Communications, Vol. 374, No. 2, 2008, pp. 378-381. doi:10.1016/j.bbrc.2008.07.030

[17] M. W. Schäffer, S. S. Roy, S. Mukherjee, et al., "Qualitative and Quantitative Analysis of Retinol, Retinyl Esters, Tocopherols and Selected Carotenoids Out of Various Internal Organs from Different Species by HPLC," Analytical Methods, Vol. 2, No. 9, 2010, pp. 1320-1332. doi:10.1039/c0ay00288g

[18] A. Matsumoto, H. Mizukami, S. Mizuno, et al., "BetaCryptoxanthin, a Novel Natural RAR Ligand, Induces ATP-Binding Cassette Transporters in Macrophages," Biochemical Pharmacology, Vol. 74, No. 2, 2007, pp. 256-264. doi:10.1016/j.bcp.2007.04.014

[19] J. E Dowling and G. Wald, "The Biological Function of Vitamin A Acid," Proceedings of the National Academy of Sciences USA, Vol. 46, No. 5, 1960, pp. 587-608. doi:10.1073/pnas.46.5.587

[20] R. Ziegler, S. Mayne and C. Swanson, "Nutrition and Lung Cancer," Cancer Causes Control, Vol. 7, No. 1, 1996, pp. 157-177. doi:10.1007/BF00115646

[21] The Alpha-Tocopherol, Beta-Carotene Cancer Prevention Study Group, "The Effect of Vitamin E and Beta Carotene on the Incidence of Lung Cancer and Other Cancers in Male Smokers," New England Journal of Medicine, Vol. 330, No. 15, 1994, pp. 1029-1035. doi:10.1056/NEJM199404143301501

[22] G. S. Omenn, G. E. Goodman, M. D. Thornquist, et al., "Effects of a Combination of $\beta$-Carotene and Vitamin A on Lung Cancer and Cardiovascular Disease," New England Journal of Medicine, Vol. 334, 1996, pp. 1150-1155. doi:10.1056/NEJM199605023341802

[23] A. Morabia, A. Sorenson, S. K. Kumanyika and H. Abbey, "Vitamin A, Cigarette Smoking and Airway Obstruction," American Review of Respiration Disease, Vol. 140, No. 5, 1989, pp. 1312-1316. doi:10.1164/ajrccm/140.5.1312

[24] A. Morabia, M. J. Menkes, G. W. Comstock and M. S. Tockman, "Serum Retinol and Airway Obstruction," American Journal of Epidemiology, Vol. 132, No. 1, 1990, pp. 77-82.

[25] J. Lorenz and H. K Biesalski, "Vitamin A-Mangel und Bronchialkarzinom: Perspektiven der Chemoprevention bronchialer Tumoren," Pneumologie, Vol. 47, No. 12, 1993, pp. 657-665.
[26] J. R. Mata, N. L. Mata and A. T. C. Tsin, "Substrate Specificity of Retinyl Ester Hydrolase Activity in Retinal Pigment Epithelium," Journal of Lipid Research, Vol. 39, 1998, pp. 604-612.

[27] T. E. Edes, S. M. Kwan, C. S. Buckley and W. H. Thornton, "Tissue Vitamin A Repletion Is Impaired by Exposure to Carcinogen," International Journal of Cancer, Vol. 50, No. 1, 1992, pp. 99-102. doi:10.1002/ijc.2910500120

[28] T. E. Edes and D. S. Gysbers, "Carcinogen-Induced Tissue Vitamin A Depletion. Potential Protective Advantages of $\beta$-Carotene," Annals of the New York Academy of Sciences, Vol. 686, 1993, pp. 203-212. doi:10.1111/j.1749-6632.1993.tb39176.x

[29] R. Kawaguchi, J. Yu, J. Honda, et al., “A Membrane Receptor for Retinol Binding Protein Mediates Cellular Uptake of Vitamin A," Science, Vol. 315, No. 5831, 2007, pp. 820-825. doi:10.1126/science. 1136244

[30] H. K. Biesalski, R. Reifen, F. Pürst and M. Edris, "Retinyl Palmitate Supplementation by Inhalation of an Aerosol Improves Vitamin A Status of Preschool Children in Gondar (Ethiopia)," British Journal of Nutrition, Vol. 82, No. 3, 1999, pp. 179-182.

[31] M. Kohlhäufl, K. Häussinger, F. Stanzel, et al., "Inhalation of Aerosolized Vitamin A: Reversibility of Metaplasia and Dysplasia of Human Respiratory Epithelia a Prospective Pilot Study," European Journal of Medical Research, Vol. 7, No. 2, 2002, pp. 72-78.

[32] M. Frankenberger, I. Heimbeck, W. Möller, et al., "Inhaled All-Trans Retinoic Acid in an Individual with Severe Emphysema," European Respiratory Journal, Vol. 34, No. 6, 2009, pp. 1487-1489. doi:10.1183/09031936.00105309

[33] S. Van Eijl, E. Mortaz, C. Versluis, F. P. Nijkamp, G. Folkerts and N. Bloksma, "A Low Vitamin A Status Increases the Susceptibility to Cigarette Smoke-Induced Lung Emphysema in C57BL/6J Mice," Journal of Physiology and Pharmacology, Vol. 62, No. 2, 2011, pp. 175182.

[34] S. Mahabir, K. Schendel, Y. Q. Dong, S. L. Barrera, M. R. Spitz and M. R. Forman, "Dietary $\alpha-, \beta$-, $\gamma$ - and $\delta$-Tocopherols in Lung Cancer Risk," International Journal of Cancer, Vol. 123, No. 5, 2008, pp. 1173-1180. doi:10.1002/ijc.23649

[35] J. L. Napoli, A. M. McCormick, B. O’Meara and E. A. Dratz, "Vitamin A Metabolism: Alpha-Tocopherol Modulates Tissue Retinol Levels in Vivo, and Retinyl Palmitate Hydrolysis in Vitro," Archives of Biochemistry and Biophysics, Vol. 230, No. 1, 1984, pp. 194-202. doi:10.1016/0003-9861(84)90100-0

[36] J. M. Zingg, "Modulation of Signal Transduction by Vitamin E," Molecular Aspects of Medicine, Vol. 28, No. 5-6, 2007, pp. 481-506. doi:10.1016/j.mam.2006.12.009

[37] E. Reiter, Q. Jiang and S. Christen, "Anti-Inflammatory Properties of $\alpha$ - and $\gamma$-Tocopherol," Molecular Aspects of Medicine, Vol. 28, No. 5-6, 2007, pp. 668-691. doi:10.1016/j.mam.2007.01.003

[38] Q. Jiang, I. Elson-Schwab, C. Courtemanche and B. N. Ames, "Gamma-Tocopherol and Its Major Metabolite, in Contrast to Alpha-Tocopherol, Inhibit Cyclooxygenase Activity in Macrophages and Epithelial Cells," Proceed- 
ings of the National Academy of Sciences of the United States of America, Vol. 97, No. 21, 2000, pp. 11494-11499. doi:10.1073/pnas.200357097

[39] J. Roca-Ferrer, L. Pujols, C. Agusti, et al.., "Cyclooxigenase-2 Levels Are Increased in the Lung Tissue and Bronchial Tumors of Patients with Chronic Obstructive Pulmonary Disease," Archivos de Bronconeumología, Vol. 47, No. 12, 2011, pp. 584-589. doi:10.1016/j.arbres.2011.05.015

[40] C. M. Greene, D. Waters, R. M. Clark, J. H. Contois and M. L. Fernandez, "Plasma LDL and HDL Characteristics and Carotenoid Content Are Positively Influenced by Egg Consumption in an Elderly Population," Nutrition \& $\mathrm{Me}$ - tabolism, Vol. 3, No. 6, 2006, pp. 6-14. doi:10.1186/1743-7075-3-6

[41] B. Li, P. Vachali, J. M. Frederick and P. S. Bernstein, "Identification of StARD3 as a Lutein-Binding Protein in the Macula of the Primate Retina," Biochemistry, Vol. 50, No. 13, 2011, pp. 2541-2549. doi:10.1021/bi101906y

[42] H. Watari, F. Arakane, C. Moog-Lutz, et al.., "MLN64 Contains a Domain with Homology to the Steroidogenic Acute Regulatory Protein (StAR) that Stimulates Steroidogenesis," Proceedings of the National Academy of Sciences of the United States of America, Vol. 94, No. 16, 1997, pp. 8462-8467. 